\title{
Ocorrência e mapeamento de cisticercose, fasciolose e hidatidose em bovinos e ovinos na região sul do Rio Grande do Sul (2018-2019) e importância em saúde pública
}

\author{
Occurrence and mapping of cysticercosis, fasciolosis and hydatidosis in cattle and sheep in the \\ southern region of Rio Grande do Sul (2018-2019) and importance in public health \\ Presencia y mapeo de cisticercosis, fasciolosis e hidatidosis en bovinos y ovinos en la región sur de \\ Rio Grande do Sul (2018-2019) e importancia en salud pública
}

Recebido: 13/01/2021 | Revisado: 15/01/2021 | Aceito: 16/01/2021 | Publicado: 19/01/2021

Diego Feijó Polvora
ORCID: https://orcid.org/ 0000-0001-6177-0242
Universidade Federal de Pelotas, Brasil
E-mail: vetdiegopolvora@ gmail.com
Kaline da Silveira Timm
ORCID: https://orcid.org/0000-0003-0754-5196
Universidade Federal de Pelotas, Brasil
E-mail: kalinetimm@gmail.com
Marina Fucolo dos Santos
ORCID: https://orcid.org/ 0000-0002-6852-3663
Universidade Federal de Pelotas, Brasil
E-mail: marinafucolo26@gmail.com
Pamela Aristimunho Sedrez
Pamela
ORCID: https://orcid.org/ 0000-0002-8739-190X
Universidade Federal de Pelotas, Brasil
E-mail: pamelaas.vet@gmail.com
Iuri Vladimir Pioly Marmitt
ORCID: https://orcid.org/ 0000-0002-5039-0161
Universidade Federal de Pelotas, Brasil
E-mail: iurihrs@hotmail.com
Fábio Raphael Pascoti Bruh
ORCID: https://orcid.org/ 0000-0002-4191-965X
Universidade Federal de Pelotas, Brasil
E-mail: fabio_rpb@yahoo.com.br
Rodrigo Casquero Cunha
ORCID: https://orcid.org/ 0000-0002-0767-4097
Universidade Federal de Pelotas, Brasil
E-mail: leandro.nizoli@gmail.com

\begin{abstract}
Resumo
A responsabilidade do setor agropecuário em produzir alimentos de forma eficaz busca também por atender essa demanda de forma sustentável gerando produtos seguros ao consumidor final. Cisticercose, fasciolose e hidatidose são zoonoses. A infecção humana ocorre pela ingestão de alimentos mal passados ou mal lavados contendo formas infectantes destes parasitos, podendo causar diversos prejuízos à saúde dos acometidos. $\mathrm{O}$ objetivo deste trabalho foi estabelecer a ocorrência destas três enfermidades em ovinos e bovinos com origem em municípios da área de abrangência da $12^{\mathrm{a}}$ Supervisão Regional do Departamento de Inspeção de Produtos de Origem Animal (DIPOA) da SEAPDR-RS, abatidos sob inspeção estadual no período de janeiro de 2018 a dezembro de 2019. Neste período foram abatidos o total de 227.946 bovinos, sendo 113.907 em 2018 e 114.039 em 2019, contabilizados animais de diversas categorias de idade. A taxa média de ocorrência considerando o período foi de $0,75 \%$ para cisticercose, $49,14 \%$ para fasciolose e 16,22\% para hidatidose. O número total de ovinos abatidos foi 45.695, dos quais, 23.746 no ano de 2018 e 21.949 no ano de 2019. As ocorrências das doenças nesta espécie, considerando a média para o período, foram $0,86 \%$ para cisticercose, $0,63 \%$ para fasciolose e $11,62 \%$ para hidatidose. Conclui-se que, neste período de estudo, a fasciolose apresentou a maior ocorrência em bovinos e a hidatidose em ovinos. Os dados avaliados neste artigo servem como ferramenta para ações em saúde pública e evidenciam a importância dos Serviços de Inspeção Oficial e do profissional Médico Veterinário na obtenção de alimentos seguros.
\end{abstract}


Palavras-chave: Prevalência; Saúde-única; Parasitoses; Inspeção.

\begin{abstract}
The responsibility of the agricultural sector to produce food effectively also seeks to meet this demand in a sustainable manner, generating safe products for the final consumer. Cysticercosis, fasciolosis and hydatidosis are zoonoses. Human infection occurs due to the ingestion of rare or poorly washed foods containing infectious forms of these parasites, which can cause several damages to the health of those affected. The objective of this work was to establish the occurrence of these three diseases in sheep and cattle originating in municipalities in the area covered by the 12th Regional Supervision of the Department of Inspection of Products of Animal Origin (DIPOA) of SEAPDR-RS, slaughtered under state inspection in the period from January 2018 to December 2019. In this period, a total of 227,946 cattle were slaughtered, 113,907 in 2018 and 114,039 in 2019, counting animals of different age categories. The average rate of occurrence considering the period was $0.75 \%$ for cysticercosis, $49.14 \%$ for fasciolosis and $16.22 \%$ for hydatidosis. The total number of slaughtered sheep was 45,695, of which, 23,746 in 2018 and 21,949 in 2019. The occurrences of diseases in this species, considering the average for the period, were $0.86 \%$ for cysticercosis, $0.63 \%$ for fasciolosis and $11.62 \%$ for hydatidosis. It is concluded that, in this study period, fasciolosis presented the highest occurrence in cattle and hydatidosis in sheep. The data evaluated in this article serve as a tool for public health actions and show the importance of the Official Inspection Services and the veterinary doctor in obtaining safe food.
\end{abstract}

Keywords: Prevalence; Unique health; Parasitoses; Inspection.

\begin{abstract}
Resumen
La responsabilidad del sector agropecuario de producir alimentos de manera efectiva también busca satisfacer esta demanda de manera sustentable, generando productos seguros para el consumidor final. La cisticercosis, la fasciolosis y la hidatidosis son zoonosis. La infección humana se produce por la ingestión de alimentos raros o mal lavados que contienen formas infecciosas de estos parásitos, que pueden causar varios daños a la salud de los afectados. El objetivo de este trabajo fue establecer la ocurrencia de estas tres enfermedades en ovinos y bovinos originarios de municipios del área comprendida por la XII Supervisión Regional del Departamento de Inspección de Productos de Origen Animal (DIPOA) de la SEAPDR-RS, sacrificados bajo inspección estatal en el período de enero de 2018 a diciembre de 2019. En este período se sacrificaron un total de 227,946 bovinos, 113,907 en 2018 y 114,039 en 2019, contando animales de diferentes categorías de edad. La tasa promedio de ocurrencia considerando el período fue de 0,75\% para cisticercosis, $49,14 \%$ para fasciolosis y 16,22\% para hidatidosis. El total de ovinos sacrificados fue de 45.695, de los cuales, 23.746 en 2018 y 21.949 en 2019. La ocurrencia de enfermedades en esta especie, considerando el promedio del período, fue del 0,86\% para cisticercosis, 0,63\%. para fasciolosis y 11,62\% para hidatidosis. Se concluye que, en este período de estudio, la fasciolosis presentó la mayor ocurrencia en bovinos y la hidatidosis en ovinos. Los datos evaluados en este artículo sirven como herramienta para las acciones de salud pública y muestran la importancia de los Servicios Oficiales de Inspección y del médico veterinario en la obtención de alimentos seguros.
\end{abstract}

Palabras clave: Prevalencia; Salud única; Parasitosis; Inspección.

\title{
1. Introdução
}

O constante aumento da população mundial nas últimas décadas demonstra a necessidade por uma maior demanda na produção de alimentos. É requerida a busca por uma produção sustentável, saudável e segura por grande parte dos consumidores, sendo também, o intuito de muitos profissionais envolvidos no setor produtivo. A agropecuária, sendo o setor primário de toda a matéria alimentícia, tende a ser o principal foco quando o assunto é garantir qualidade e segurança nos alimentos que chegam ao consumidor final (Maluf et al., 2015).

Atualmente, o Médico Veterinário destaca-se como profissional que assegura a cadeia produtiva devido sua interdisciplinaridade, e isso permite não somente a devida importância nas áreas de saúde animal, como acerca da área de saúde pública com a prevenção e promoção à saúde humana, onde o principal tema de atuação são as zoonoses, garantindo assim, o alimento seguro (Armelin \& Cunha, 2016). Dentre as diversas doenças transmitidas por contato com animais, existem as de origem parasitária que podem ser transmitidas pela carne e seus derivados, caso estes apresentem contaminação. Neste contexto, observa-se a importância da inspeção no abate de animais de produção a fim de detectar doenças, principalmente aquelas com potencial infectante, na carne e nos órgãos de consumo (Zanella, 2016). 
Rossi et al., (2014) destacam que as zoonoses causadas por agentes parasitários ainda são muito comuns no Brasil. Alguns dos municípios brasileiros chegam a apresentar uma soroprevalência de 13,8\% para cisticercose (Guarda et al., 2018). A ingestão de cistos como os Cysticercus celullosae e Cysticercus bovis em carnes cruas ou cujo preparo é dado por mal passadas gera a ocorrência da teníase humana pelos parasitos Taenia solium e Taenia saginata, respectivamente (Zanella, 2016). Os sinais clínicos causados pela teníase são desde dor abdominal, náuseas, diarreia, até perda de peso ou prisão de ventre (Pfuetzenreiter \& Pires, 2000).

A infecção humana também pode ocorrer por meio da ingestão de formas infectantes quando presente em vegetais contaminados. Esta transmissão acontece em decorrência da defecação de animais carnívoros próximo aos cultivos que, previamente, tenham consumido vísceras ou carcaças de abates clandestinos ou animais mortos que estavam infectados por estes parasitos, atuando como hospedeiros. Como exemplo, tem-se a hidatidose humana, que ocorre após a ingestão de vegetais contaminados com ovos do Echinococcus granulosus, parasita intestinal de cães e que, durante o consumo de vísceras contaminadas com cistos hidáticos, acabam por serem infectados (Kloetzel \& Pereira, 1992).

Hábitos alimentares humanos, quando associados à presença de parasitas em rebanhos, são fatores que influenciam para ocorrência de zoonoses. O consumo de agrião silvestre e água contaminada são as principais razões de contaminação de fasciolose em humanos. Esta doença, causada pelo trematódeo Fasciola hepatica, ocorre nos hospedeiros definitivos (bovinos, ovinos, animais silvestres e acidentalmente o homem) pelo consumo de vegetais contendo a forma infectante, denominada metacercária. Nos humanos, a doença apresenta forma aguda pela manifestação de sintomas de febre, dor abdominal e inchaço do fígado. Já na forma crônica, ocorre a inflamação hepática e pode causar perda de peso, febre, acúmulo de líquido no abdômen, anemia, tonturas e falta de ar (Silva et al., 2008).

A atuação do Médico Veterinário é importante tanto na inspeção destes produtos, do abate ao fornecimento, quanto na promoção de saúde animal, garantindo a sanidade dos rebanhos a fim de evitar a ocorrência de doenças, orientando produtores, identificando fatores predisponentes ao seu surgimento e propondo metodologias de tratamento. A análise de dados provenientes da Inspeção Oficial em frigoríficos é uma das formas de identificar a ocorrência destas doenças em animais de produção da região, demonstrando assim, quais as zonas de maior prevalência para tais enfermidades. Estes dados confirmam sua importância pois, por meio da análise destes, é possível alertar o perigo ao consumir carne e derivados cuja origem do produto é de abates clandestinos provenientes dessas áreas.

O objetivo geral deste trabalho foi avaliar a ocorrência de cisticercose, fasciolose e hidatidose em bovinos e ovinos oriundos dos municípios da área de abrangência da $12^{\circ}$ Supervisão Regional do Departamento de Inspeção de Produtos de Origem Animal (DIPOA) da Secretária de Agricultura, Pecuária e Desenvolvimento Rural do Estado do Rio Grande do Sul (SEAPDR-RS), abatidos sob inspeção estadual no período de janeiro de 2018 a dezembro de 2019, criando uma distribuição espacial a fim de identificar as áreas de maior ocorrência dessas doenças nos rebanhos de produção, utilizando os dados como meios de prevenção de zoonoses parasitárias na saúde pública.

\section{Metodologia}

Os dados utilizados no presente trabalho foram obtidos do Setor de Epidemiologia e Estatística da SEAPDR-RS. Foram solicitados dados mensais de abates de bovinos e ovinos, de todas as categorias, abatidos em estabelecimentos sob inspeção estadual (DIPOA-RS) no período de janeiro de 2018 a dezembro de 2019. Para realização deste trabalho, foram selecionados somente os dados de animais que são de origem de 20 munícipios sob área de atuação da $12^{\mathrm{a}}$ Supervisão (Figura 1), e abatidos em estabelecimentos da mesma supervisão. 
Figura 1. Mapa do Rio Grande do Sul com destaque dos municípios de abrangência da $12^{\circ}$ Supervisão Regional do Departamento de Inspeção de Produtos de Origem Animal (DIPOA) da Secretária de Agricultura, Pecuária e Desenvolvimento Rural do Estado do Rio Grande do Sul (SEAPDR-RS), utilizados no estudo.

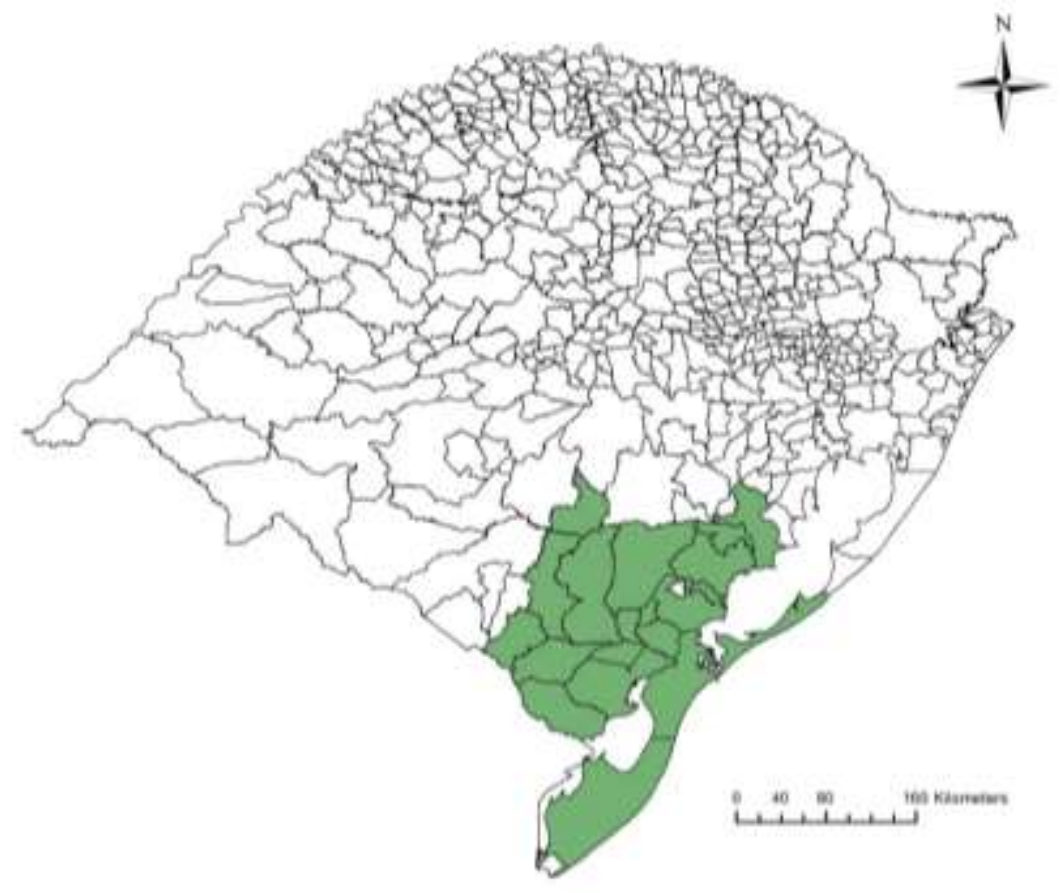

Fonte: Autores.

As carcaças e vísceras dos animais abatidos são inspecionadas macroscopicamente pelo Médico Veterinário do sistema de inspeção estadual e auxiliares de inspeção, no momento denominado inspeção pós mortem. Na maioria das vezes as lesões encontradas no momento desta inspeção determinam a doença, e o Médico Veterinário pode tomar as providências quanto ao destino da peça afetada. As lesões de cisticercose caracterizam-se por vesículas cheias de líquido contendo um único excoléx invaginado encontradas em meio à fibras musculares, as lesões de fasciolose são caracterizadas pela fibrose de ductos hepáticos e também presença de formas adultas de Fasciola hepatica, na hidatidose as lesões são a presença de um ou mais cistos cheios de líquidos e tamanhos variados, contendo escólices invaginados livres ou em cachos cercados por vesículas prolígeras, essas são encontrados em órgãos como pulmão e fígado (McGAVIN et al., 2013; Monteiro, 2007). Este trabalho é de natureza quantitativa. Os dados referentes a ocorrência de cisticercose, fasciolose e hidatidose são planilhados e alocados no sistema ficando disponíveis no Setor de Epidemiologia da SEAPDR-RS. Foram feitas análises descritivas das variáveis levantadas nos sistemas de informação, como a incidência da Cisticercose, Hidatidose e Fasciolose em bovinos e ovinos, faixa etária dos animais e seu município de criação. Além disso, a taxa de incidência (total positivos/total abatido) das diferentes doenças foi comparada entre os municípios por meio da construção de mapas descritivos por área, usando os sistemas de informação geográficos (SIG), por meio do programa estatístico QGis 3.14.1 (Pereira et al., 2018).

\section{Resultados e Discussão}

No período de janeiro de 2018 a dezembro de 2019, os abates de bovinos e ovinos com origem nos munícipios analisados somaram, entre as espécies, 273.641 animais, sendo, deste total, 227.946 (83,30\%) da espécie bovina. Os municípios de Canguçu, Santa Vitória do Palmar e São Lourenço do Sul foram os que mais enviaram bovinos para abate, nos 
dois anos seguidos, registrando mais de 10.000 bovinos (cada um) enviados para abate em frigoríficos da mesma região. O município de São José do Norte não alcançou a marca de 500 bovinos enviados nos dois anos avaliados. Na Figura 2 é demonstrado o total de bovinos abatidos por município de origem nos dois anos respectivamente.

Destaca-se as categorias de animais abatidos no ano de 2018, 54,71\% dos animais abatidos estavam na categoria “acima de 36 meses", $21,26 \%$ de "25 a 36 meses", 22,27\% na categoria de "13 a 24 meses" e somente 1,75\% na categoria de "0 a 12 meses". O levantamento de dados de 2019 demonstrou porcentagens bem semelhantes com 54,36\% de bovinos "acima de 36 meses", 21,13\% de "25 a 36 meses", 21,78\% de "13 a 24 meses" e 2,73\% de "0 a 12 meses" (Figura 3).

A região Sul do Rio Grande do Sul destaca-se por ser uma das regiões que mais origina bovinos para abate. No primeiro semestre de 2017 a região foi origem de mais de 45.000 bovinos destinados a abate entre todos os níveis de inspeção (Municipal, Estadual e Federal). Os dados de abate do referido semestre corroboram com os encontrados neste levantamento onde a maior proporção foi de animais na categoria de "acima de 36 meses". A menor proporção foi de animais da categoria de "0 a 12 meses", ficando as outras duas categorias com proporções intermediarias (SEAPI-RS, 2017).

Figura 2. Total de bovinos abatidos sob inspeção estadual (DIPOA-RS), por município de origem na região sul do Rio Grande do Sul, 2018 a 2019.

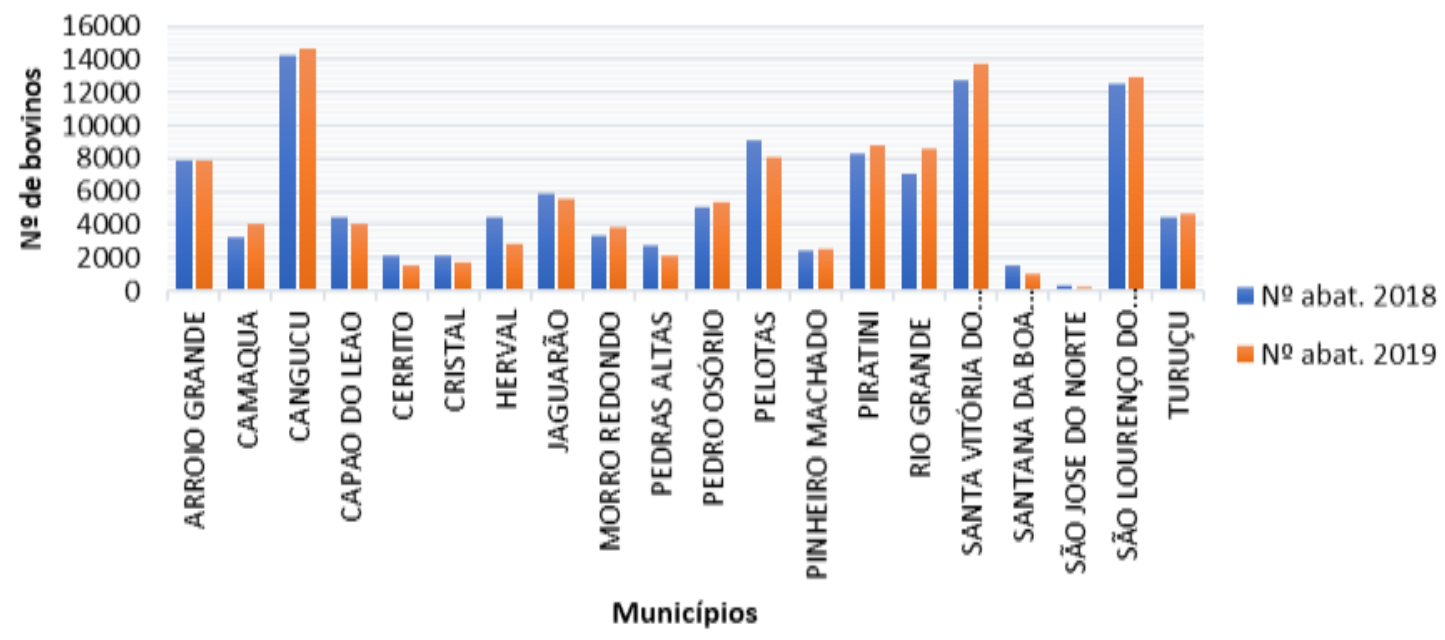

Fonte: Autores.

Figura 3. Porcentagem de categorias de bovinos abatidos sob inspeção estadual (DIPOA-RS), por município de origem na região sul do Rio Grande do Sul, 2018 a 2019.

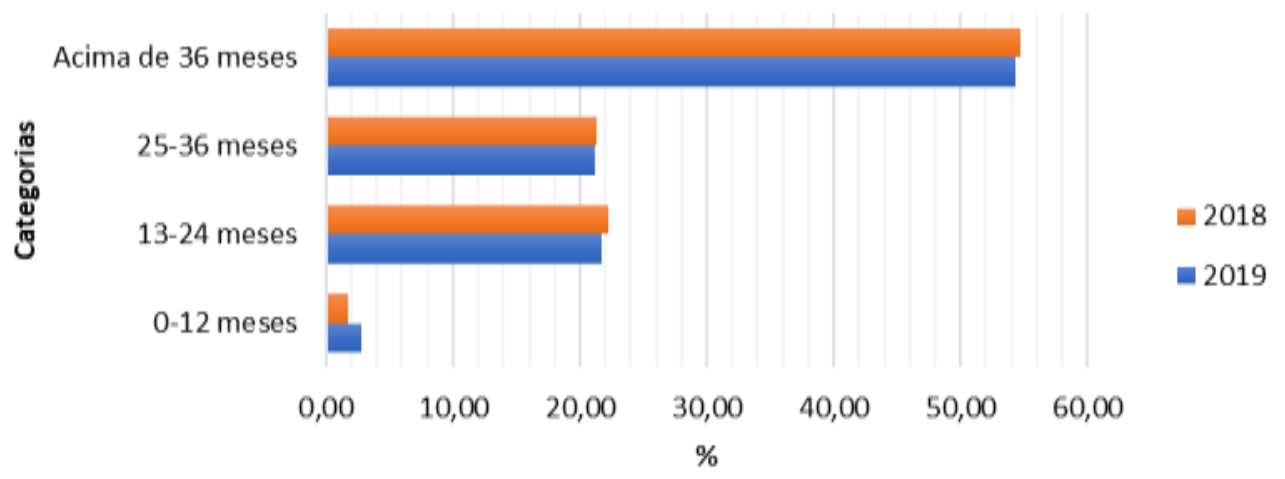

Fonte: Autores. 
Entre todos os municípios, um total de 113.907 bovinos foram abatidos no ano de 2018. Deste total, 949 (0,83\%) bovinos estavam afetados por cisticercose, 36.466 (32,01\%) por fasciolose e 19.535 (17,15\%) por hidatidose. Não muito diferente, no ano de 2019, foram abatidos 114.039, dos quais 774 (0,68\%) bovinos estavam afetados por cisticercose, 39.107 $(34,29 \%)$ por fasciolose e $17.433(15,29 \%)$ por hidatidose. As distribuições de incidências destes dados estão representadas de forma ilustrada na Figura 4.

A análise mensal de ocorrência de doenças demonstrou picos na ocorrência de fasciolose em bovinos no mês de maio em ambos os anos, no mês de setembro em 2018 e novembro em 2019. A ocorrência de hidatidose nesta espécie se manteve entre 10 e 30\% durantes o período analisado, apresentando poucas variações. A incidência de cisticercose foi estável, apresentando baixos números no período (Figura 5).

Figura 4. Mapas de proporção de incidência de zoonoses parasitárias em bovinos abatidos sob inspeção estadual (DIPOA-RS), por município de origem na região sul do Rio Grande do Sul, nos anos de 2018 e 2019.

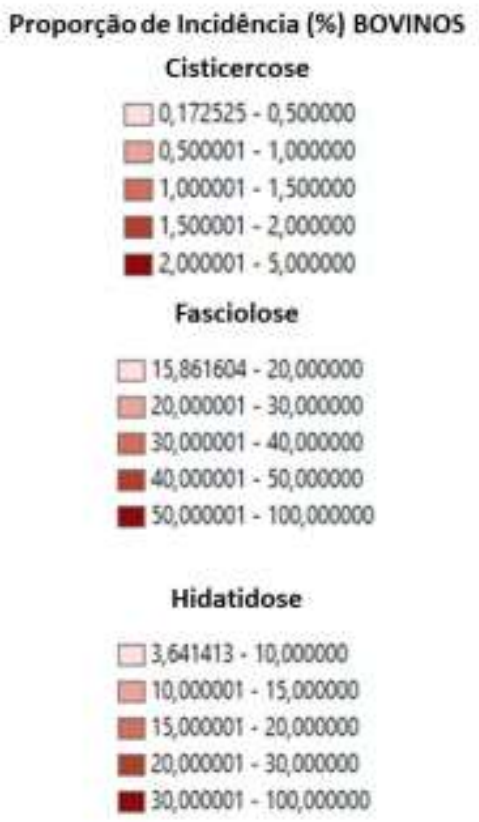

2018
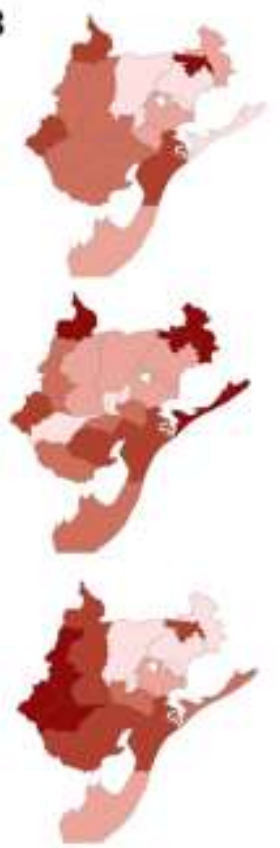

2019
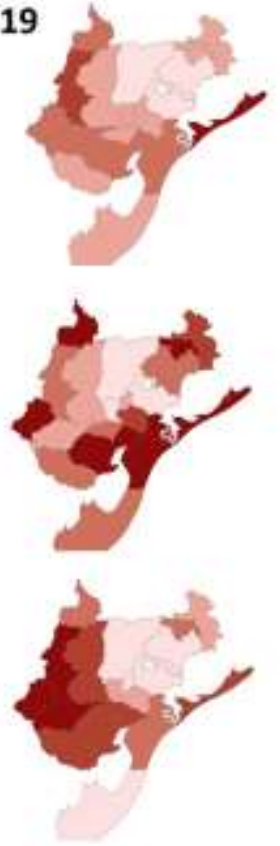

Fonte: Autores. 
Figura 5. Incidência mensal de cisticercose, fasciolose e hidatidose em bovinos abatidos sob inspeção estadual (DIPOA-RS), por município de origem, na região sul do Rio Grande do Sul, nos anos de 2018 e 2019.

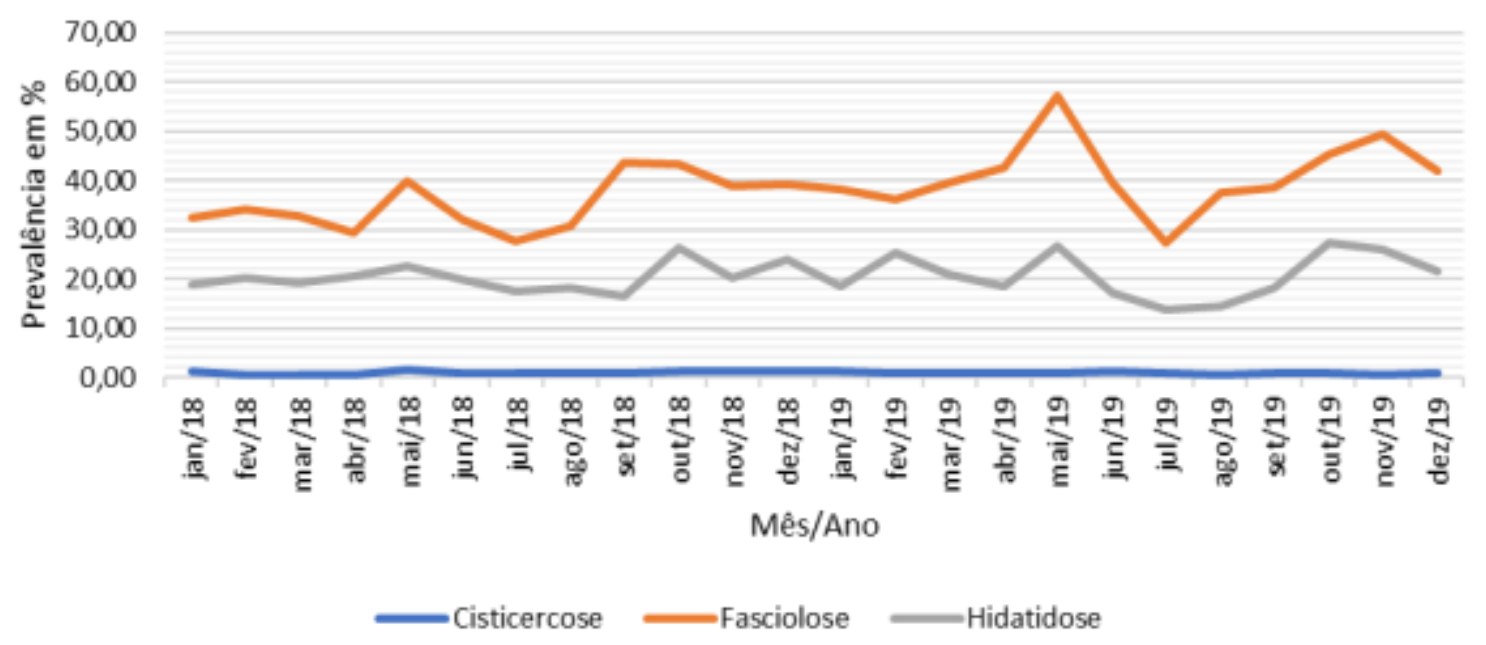

Fonte: Autores.

Conforme a ilustração nos mapas pode-se observar que a proporção de incidência de cisticercose em bovinos foi bem variável entre os anos analisados e destaca-se uma ocorrência maior no município de Cristal em 2018 e em São José do Norte em 2019.

Com relação à espécie ovina, um total de 45.695 animais (16,70\%) foram abatidos no período e, neste caso, é possível observar que o munícipio que se destacou como maior originário de ovinos para abate foi São Lourenço do Sul, com mais de 7.000 ovinos a cada ano avaliado. Os demais municípios ficaram abaixo de 3.000 ovinos enviados para abate. Na figura 6 pode-se observar o total de ovinos abatidos por munícipio de origem nos dois anos, respectivamente.

Destaca-se que os ovinos são separados em apenas duas categorias ao abate, diferentemente dos bovinos. As categorias são: "0 a 12 meses" e "acima de 12 meses". No ano de 2018, 52,79\% dos ovinos abatidos foram da categoria "acima de 12 meses" e 47,21\% foram da categoria de "0 a 12 meses". Em 2019, 55,66\% dos ovinos abatidos pertenciam à categoria "Mais de 12 meses" e 44,34\% à categoria de "0 a 12 meses" (Figura 7). 
Figura 6. Total de ovinos abatidos sob inspeção estadual (DIPOA-RS), por município de origem na região sul do Rio Grande do Sul, durante os anos de 2018 e 2019.

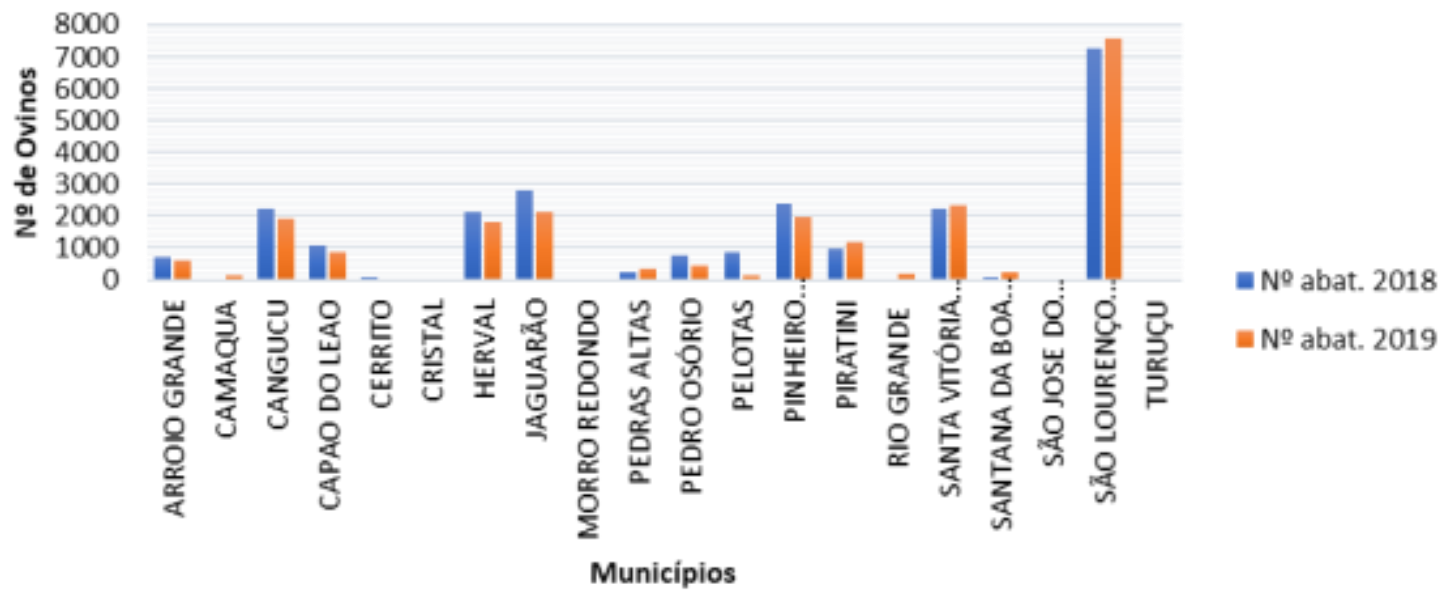

Fonte: Autores

Figura 7. Porcentagem de categorias de ovinos abatidos sob inspeção estadual (DIPOA-RS), de municípios de origem na região sul do Rio Grande do Sul, durante os anos de 2018 e 2019.

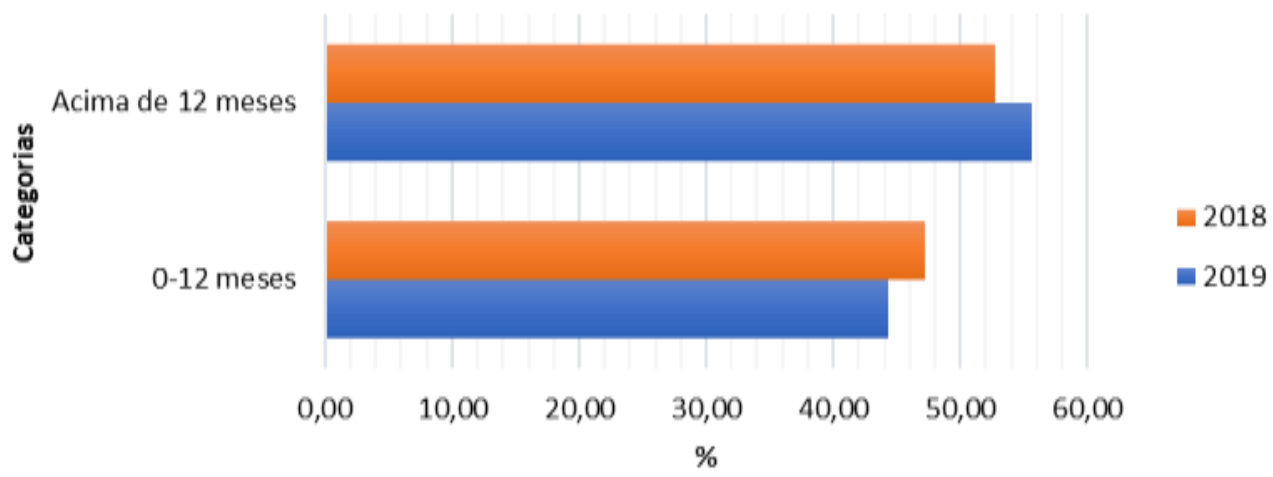

Fonte: Autores.

O número total de animais abatidos da espécie ovina no ano de 2018 foi 23.746, destes, 78 (0,33\%) estavam afetados por cisticercose, 203 (0,85\%) por fasciolose e 2457 (10,35\%) para hidatidose. No ano de 2019 foram 21.949 ovinos abatidos, $305(1,39 \%)$ afetados para cisticercose, 91 (0,41\%) por fasciolose e 2831 (12,90\%) por hidatidose (Figura 8). Nesta espécie, a maior incidência foi de hidatidose, seguida de faciolose e cisticercose, ambas apresentaram picos de dezembro de 2018 a abril de 2019 (Figura 9). 
Figura 8. Mapas de proporção de incidência de zoonoses parasitárias em ovinos abatidos sob inspeção estadual (DIPOA-RS), por município de origem da região sul do Rio Grande do Sul, durante os anos de 2018 e 2019.
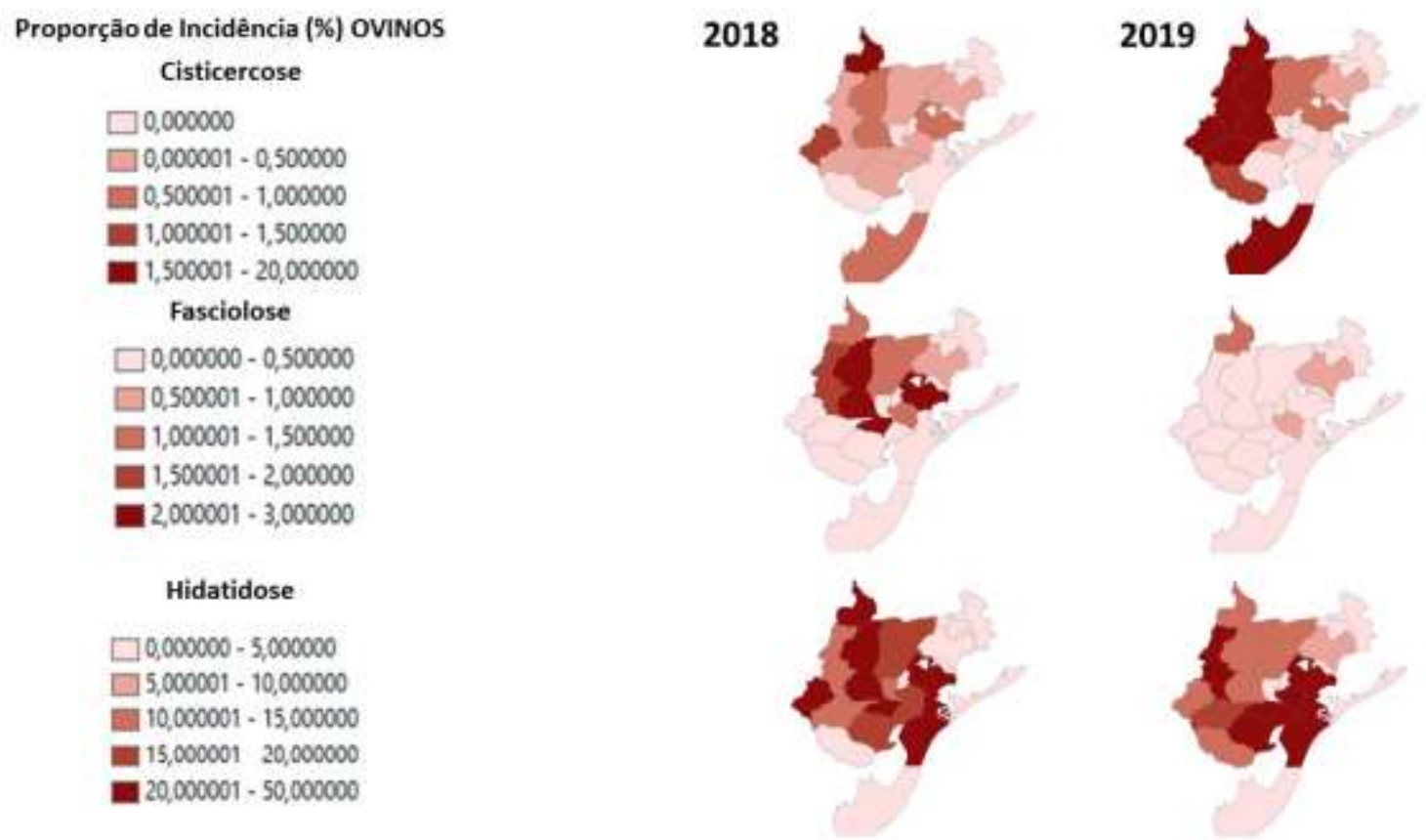

Fonte: Autores.

Figura 9. Prevalência mensal de cisticercose, fasciolose e hidatidose em ovinos abatidos sob inspeção estadual (DIPOA-RS), por município de origem da região sul do Rio Grande do Sul, durante os anos de 2018 e 2019.

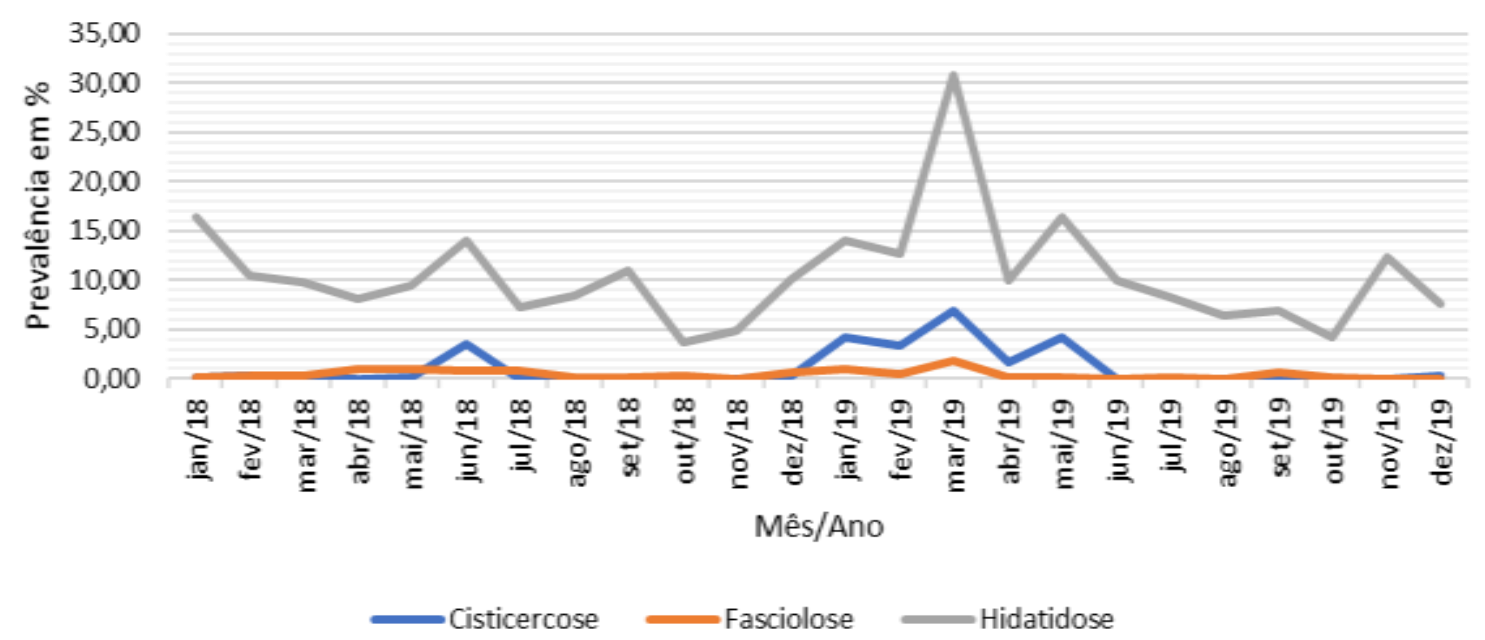

Fonte: Autores.

Comparando-se entre os anos a prevalência de cisticercose em ovinos se mostrou maior no ano de 2019 com proporção maior que 1,5\% nos munícipios de Herval, Pinheiro Machado, Pedras Altas, Piratini e Santana da Boa Vista e Santa Vitória do Palmar. No ano de 2018 essa proporção foi avaliada somente no município de Santana da Boa Vista. A maior incidência de fasciolose foi registrada no ano de 2018 nos municípios Piratini, Pelotas e Pedro Osório. Entre as doenças de ovinos a hidatidose apresentou a maior incidência (maior de 15\%) na maioria dos municípios e ambos os anos. 
A hidatidose, forma larval do cestodeo Echinococcus granulosus, é uma zoonose de grande importância em saúde pública. A presença de cães errantes em municípios com rebanhos que apresentam alta prevalência torna-se um fator de importância na transmissão da doença para seres humanos. Na região da campanha, no Rio Grande do Sul, (Hoffmann et al., 2001) realizaram uma pesquisa utilizando cães errantes do município de Dom Pedrito-RS, constando que aproximadamente 10\% dos animais amostrados estavam positivos para E. granulosus. A infecção de cães se dá através do consumo de vísceras de animais mortos naturalmente, de abates caseiros ou em decorrência do crime de abigeato. Em um estudo realizado em um frigorifico de inspeção estadual, com ovinos provenientes da região Sul e fronteiras com Uruguai e Argentina, foi detectada frequência de 19,8\% de cistos hidáticos (Freitas et al., 2013).

Em bovinos dos municípios de Pelotas, Canguçu, São Lourenço, Arroio Grande e Capão do Leão existem relatos de 19,96 \% de média de incidência para hidatidose nos anos avaliados (2013, 2014, 2015, 2016) (Alberti et al., 2018). Os dados se mostram semelhantes quando avaliados com uma maior abrangência, como demonstram (Bica et al., 2014), que encontraram a ocorrência de 20,26 \% de bovinos afetados por hidatidose nos abates da região de Pelotas. Neste trabalho o número de bovinos afetados por hidatidose foi muito semelhante entre os anos de 2018 e 2019, mantendo-se a proporção acima de $40 \%$ para os animais com origem nos municípios de Herval, Pedras Altas e Pinheiro Machado.

Em outras regiões do Brasil foram encontradas prevalências bem menores para hidatidose em bovinos, como no estado de São Paulo, onde as prevalências encontradas para enfermidade foram de 0,46\% e 0,84\% nos anos de 2014 e 2015, respectivamente (Hubener et al., 2019). No Rio Grande do Sul, vários fatores podem estar associados a ocorrência de hidatidose na região Sul. A criação conjunta de ovinos e bovinos e a presença de cães errantes e canídeos silvestres contribuem para continuação do ciclo do parasito dentro das propriedades. O sistema de criação de ovinos é na sua maioria extensivo, o que facilita a predação e ocorrências de abigeato (Silva et al., 2013).

A proporção indicada para a fasciolose na espécie bovina foi maior em ambos os anos nos municípios banhados pela laguna Lagoa dos Patos e pela Lagoa Mirim (Arroio Grande, Pelotas, Capão do Leão, Rio Grande e São José do Norte). Estes resultados podem estar associados ao fato de que nessas regiões existem muitas áreas de banhado, gerando assim a ambiência ideal para o desenvolvimento do seus hospedeiros intermediários, caramujos do gênero Lymnea spp. (Coelho \& Lima, 2003). Os dados encontrados corroboram com Bennema et al. (2014), que detectaram uma prevalência de fasciolose entre 10 e $30 \%$ na mesma região. Nesse estudo é destacado que a maior prevalência de fasciolose está no estado do Rio Grande do Sul e ao longo da costa de Santa Catarina e Paraná.

Estudos anteriores relatam uma queda na prevalência de fasciolose em ovinos no Rio Grande do Sul (Da Cunha et al., 2007). Os dados apresentados neste trabalho ainda se mostram menores quando comparados aos dados de (Dall Asta et al., 2013), que encontrou uma prevalência de 1,33\% em ovinos da região da Campanha do Rio Grande do Sul.

A fasciolose representa um considerável impacto econômico, pois, além de afetar a reprodução e a produtividade dos animais, leva a condenação do fígado ao abate gerando prejuízos no sistema de produção como um todo (Rodrigues \& Souza, 2019). No Rio Grande do Sul as perdas por condenação podem chegar a um prejuízo de R \$ 18 milhões e o estado apresenta altas prevalências devido aos seus aspectos geográficos, assim, os dados encontrados demonstram que quanto menor a altitude maior a prevalência da doença (Rosa, 2016).

A ocorrência de cisticercose em bovinos foi a mais baixa comparada às demais doenças já encontradas em ovinos. Apesar de resultados semelhantes, em alguns momentos superou a ocorrência de fasciolose. Os dados de incidências apresentados aqui demonstram-se menores do que os encontrados por outros autores. Hubener et al. (2019) relataram uma ocorrência média de 2,41\% em dois anos analisados na espécie bovina. Em ovinos na região da Campanha foi relatado a ocorrência de 3,20\% de ovinos com cisticercose. Ainda que os resultados encontrados neste trabalho sejam baixos em relação a 
ocorrência de cisticercose, ela é prevalente nos rebanhos e representa um risco a saúde pública visto que pode-se observar sua prevalência em outras espécies de animais utilizados na produção (Acevedo-Nieto et al., 2017).

\section{Considerações Finais}

Com o presente estudo foi possível observar a ocorrência das zoonoses cisticercose, fasciolose e hidatidose na região de abrangência da $12^{\mathrm{a}}$ Supervisão Regional do Departamento de Inspeção de Produtos de Origem Animal (DIPOA) da Secretária de Agricultura, Pecuária e Desenvolvimento Rural do Estado do Rio Grande do Sul (SEAPDR-RS), região esta que compõe a metade sul do estado. Pode se observar que a fasciolose tem maior ocorrência em bovinos, seguida da hidatidose e da cisticercose. Já na espécie ovina, a hidatidose obteve maiores taxas de ocorrência, e a cisticercose e a fasciolose apresentaram valores de ocorrências menores, porém, consideráveis.

Para a realização de trabalhos futuros dados como esses podem ser melhores avaliados levando em consideração períodos mais longos de observação, fatores climáticos e sistemas de criação para a obtenção de resultados mais precisos. A utilização deste estudo pode servir como ferramenta indispensável para contribuir com epidemiologia das zoonoses e assim estabelecer ações mais efetivas em saúde pública. Também fica evidente a importância da atuação dos Serviços Oficiais de Inspeção (SIM, DIPOA, SIF) e do Médico Veterinário como agentes de saúde pública garantindo a obtenção de alimentos mais seguros ao consumidor final.

\section{Referências}

Acevedo-Nieto, E. C., Pinto, P. S. A., Silva, L. F., Guimarães-Peixoto, R. P. M., Santos, T. O., Ducas, C. T. S., \& Bevilacqua, P. D. (2017). Prevalence and risk factors for porcine cysticercosis in rural communities of eastern Minas Gerais, Brazil. Pesquisa Veterinária Brasileira, 37(9), 905-910. https://doi.org/10.1590/s0100-736x2017000900001.

Alberti, T. S., Bruhn, F. R. P., Lansini, V., Raffi, M. B., Scheid, H. V., Zamboni, R., Quevedo, L., \& Sallis, E. S. V. (2018). Occurrence of hydatidosis and cysticercosis in cattle in southern Rio Grande do Sul, Brazil, from 2013 to 2016. Pesquisa Veterinaria Brasileira, 38(10), 1918-1922. https://doi.org/10.1590/1678-5150-PVB-5865

Bennema, S. C., Scholte, R. G. C., Molento, M. B., Medeiros, C., \& Carvalho, O. dos S. (2014). Fasciola hepatica IN Bovines In Brazil: Data Availability And Spatial Distribution. Revista Do Instituto de Medicina Tropical de São Paulo, 56(1), 35-41. https://doi.org/10.1590/s0036-46652014000100005.

Bica, R. F., Brum, M. C. S., \& Copetti, M. V. (2014). Ocorrência de Cisticercose, Tuberculose e Hidatidose em Bovinos Abatidos Sob Inspeção Estadual no Rio Grande do Sul, Brasil, em 2013. 189-190. https://doi.org/10.5151/foodsci-microal-247.

Coelho, L. H. L., \& Lima, W. S. (2003). Population dynamics of Lymnaea columella and its natural infection by Fasciola hepatica in the State of Minas Gerais, Brazil . Journal of Helminthology, 77(1), 7-10. https://doi.org/10.1079/joh2002138.

Da Cunha, F. O. V., Marques, S. M. T., \& De Mattos, M. J. T. (2007). Prevalence of slaughter and liver condemnation due to Fasciola hepatica among sheep in the state of Rio Grande do Sul, Brazil 2000 and 2005. Parasitologia Latinoamericana, 62(3-4), 188-191. https://doi.org/10.4067/s071777122007000200015 .

Dall Asta, L. S., Hubert, A. R. N., Feijó, F. D., Cheuiche, Z. M. G., \& Brum, L. P. (2013). Prevalência de hidatidose, cisticercose e fasciolose em ovinos abatidos em um frigorífico da região da Campanha Riograndense. In Anais do Salão Internacional de Ensino, Pesquisa e Extensão (Vol. 3).

Freitas, L. V. De, Sawitzki, G. C., Vieira, T. P., \& Brum, L. P. (2013). Prevalência De Hidatidose Em Rebanhos Ovinos Na Região Sul Do Rio Grande Do Sul E Fronteiras Com Uruguai E Argentina. In Anais do Salão Internacional de Ensino, Pesquisa e Extensão (Vol. 1).

Guarda, K. X. da, Costa-Cruz, J. M., \& Barcelos, I. S. da C. (2018). Seroprevalence of human cysticercosis in Jataí, Goiás state, Brazil. The Brazilian Journal of Infectious Diseases, 22(2), 146-149. https://doi.org/10.1016/j.bjid.2018.01.002.

Hoffmann, A. N., Malgor, R., \& Rue, M. L. de la. (2001). Prevalência de Echinococcus granulosus (Batsch, 1786) em cães urbanos errantes do município de Dom Pedrito (RS), Brasil. Ciência Rural, 31(5), 843-847. https://doi.org/10.1590/s0103-84782001000500017.

Hubener, E., Dian, P. H. M., Belo, M. A. A., \& SOARES, V. E. (2019). Cisticercose, Faciolose E Hidatididose Em Bovinos Abatidos Na Área Centro-Oeste Do Estado De São Paulo. Ars Veterinaria, 35(3), 93. https://doi.org/10.15361/2175-0106.2019v35n3p93-99.

Kloetzel, K., \& Pereira, J. A. A. (1992). A hidatidose humana no Rio Grande do Sul (Brasil): estimativa de sua importância para a saúde pública do país. Revista Do Instituto de Medicina Tropical de São Paulo, 34(6), 549-555. https://doi.org/10.1590/S0036-46651992000600009.

Maluf, R. S., Burlandy, L., Santarelli, M., Schottz, V., \& Speranza, J. S. (2015). Nutrition-sensitive agriculture and the promotion of food and nutrition sovereignty and security in Brazil. Ciência \& Saúde Coletiva. https://doi.org/10.1590/1413-81232015208.14032014. 
Research, Society and Development, v. 10, n. 1, e39810111939, 2021

(CC BY 4.0) | ISSN 2525-3409 | DOI: http://dx.doi.org/10.33448/rsd-v10i1.11939

McGAVIN, D., Romano, N., Baldassini, M. R., Buonocore, F., Picchietti, S., Mastrolia, L., Abelli, L., Foresti, F., Oliveira, C., Foresti de Almeida-Toledo, L., Ouimet, 10, Langley-, C. C., Guillion, K.-C., Greengard, P., Snyder, S. H., Katims, J. J., Annau, Z., Bruns, R. F., Daly, J., \& Fao. (2013). Bases da Patologia em Veterinária. Hormones and Reproduction in Fishes, Amphibians, and Reptiles.

Monteiro, S. G. (2007). Parasitologia Veterinária. Livro.

Pereira, A. S., Shitsuka, D. M., Parreira, F. J., \& Shitsuka, R. (2018). Método Qualitativo, Quantitativo ou Quali-Quanti. In Metodologia da Pesquisa Científica. https://repositorio.ufsm.br/bitstream/handle/1/15824/Lic_Computacao_Metodologia-Pesquisa-Cientifica.pdf?sequence=1.

Pfuetzenreiter, M. R., \& Pires, F. D. de Á. (2000). Epidemiologia da teníase/cisticercose por Taenia solium e Taenia saginata. Ciência Rural, 30(3), 541-548. https://doi.org/10.1590/S0103-84782000000300030.

Rodrigues, H. da C., \& Souza, H. B. de. (2019). Prevalência e perdas econômicas por fasciolose em bovinos abatidos sob regime de inspeção municipal em Itaperuna, Rio de Janeiro. Pubvet, 13(1), 1-4. https://doi.org/10.31533/pubvet.v13n1a254.1-4.

Rosa, M. C. (2016). Prevalência De Fasciolose Em Bovinos Abatidos Sob Regime De Inspeção Federalno Estado Do Rio Grande Do Sul. Universidade Federal Do Rio Grande Do Sul.

Rossi, G. A. M., Hoppe, E. G. L., Martins, A. M. C. V., \& Prata, L. F. (2014). Zoonoses parasitárias veiculadas por alimentos de origem animal: revisão sobre a situação no Brasil. Arquivos Do Instituto Biológico, 81(3), 290-298. https://doi.org/10.1590/1808-1657000742012.

SEAPI-RS. (2017). Estatísticas de Proteína Animal no RS (Vol. 1, pp. 1-13).

Silva, A. P. S. P., Santos, D. V., Kohek, I., Machado, G., Hein, H. E., Vidor, A. C. M., \& Corbellini, L. G. (2013). Ovinocultura do Rio Grande do Sul: Descrição do sistema produtivo e dos principais aspectos sanitários e reprodutivos. Pesquisa Veterinaria Brasileira, 33(12), 1453-1458. https://doi.org/10.1590/S0100-736X2013001200010.

Silva, E. R. V. da, Capoani, R. Q., Ritz, R., Surian, C. R. de S., \& Neves, M. F. (2008). Fasciolose Hepática. Revista Científica Eletrônica de Medicina Veterinária, 6(11), 1-7.

Tollstadius Armelin, N., \& Almeida Cunha, J. R. (2016). O papel e a importância do médico veterinário no sistema único de saúde: uma análise à luz do direito sanitário. Cadernos Ibero-Americanos De Direito Sanitário, 5(1), 60. https://doi.org/10.17566/ciads.v5i1.245.

Zanella, J. R. C. (2016). Zoonoses emergentes e reemergentes e sua importância para saúde e produção animal. Pesquisa Agropecuária Brasileira, 51(5), 510519. https://doi.org/10.1590/s0100-204x2016000500011. 